\title{
Translational research and nursing
}

\author{
Evelin Capellari Cárnio
}

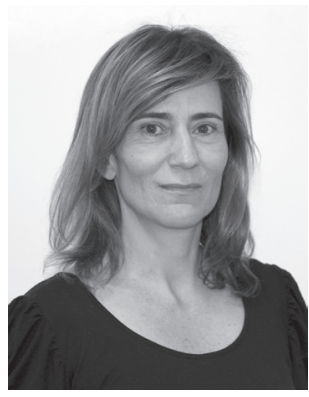

Translational research is presented in a context of transformation in scientific findings, originating in basic, clinical research laboratories or epidemiological studies and providing new clinical tools, processes or applications(1). Translational research is aimed at improving patient care and promoting public health. To reach this goal, the intent is to build a bridge that connects the research laboratory benches with the bedside. In that sense, it involves many researchers from different areas, concentrating efforts to improve populations' quality of life and longevity through their cooperation.

Nursing studies how patients respond to illnesses or adapt to changes, using data and observations deriving from clinical practice and research development. Hence, nurse scientists should identify effective strategies to accelerate translational research and develop studies in cooperation with basic and clinical researchers.

Translational research involves two-way and dynamic phases, with complex links of feedback, which are colloquially called the 3 b's $\neg$ (bench, bedside and back again) and then grouped in three phases. The first is when the original ideas emerge to be translated into clinical practice. It refers to bench research, undertaken in controlled laboratory conditions, generally involving in vitro research or animal models. In Brazil, in general, these studies are developed in biology, pharmacy, biomedicine, but rarely in nursing.

The second phase tests the clinical effectiveness of the new finding or scientific tool through comparative tests, aiming to identify the right treatment for the patient in question, generally developed by professionals involved in research and clinical practice.

In phase three, the link between clinical research and practice should be established, disseminating how the new finding can lead to advancements in care or, also, to the construction of new health policies, based on scientific knowledge produced in the two earlier phases.

It should be reminded that these three phases provide mutual support and feedback. Their focus should be maintained on the removal of barriers that hamper multidisciplinary cooperation.

\section{References}

1. PerzynskiAT. Multidisciplinary approaches to biomedical research. JAMA. 2010;304(20):2243-4.

Evelin Capellari Cárnio is Associate Editor of the Latin American Journal of Nursing, and Associate Professor of the Escola de Enfermagem de Ribeirão Preto, Universidade de São Paulo, WHO Collaborating Centre for Nursing Research Development, Brazil, e-mail carnioec@eerp.usp.br 
